\title{
A New Nonlinear Contraction Principle in Partial Metric Spaces
}

\author{
Yi Zhang ${ }^{1}$ and Jiang $\mathrm{Zhu}^{2}$ \\ ${ }^{1}$ School of Mathematics and Physics, XuZhou Institute of Technology, Xuzhou 221008, China \\ ${ }^{2}$ School of Mathematics and Statistics, Jiangsu Normal University, Xuzhou 221116, China
}

Correspondence should be addressed to Jiang Zhu; jzhuccy@163.com

Received 15 August 2016; Revised 19 October 2016; Accepted 26 October 2016

Academic Editor: Nawab Hussain

Copyright (C) 2016 Y. Zhang and J. Zhu. This is an open access article distributed under the Creative Commons Attribution License, which permits unrestricted use, distribution, and reproduction in any medium, provided the original work is properly cited.

\begin{abstract}
We present a new nonlinear contraction principle on partial metric spaces and prove the existence of common fixed point. We also give some examples to show our results and apply our results to study the existence of common bounded solution of the system of functional equations.
\end{abstract}

\section{Introduction}

There exist many generalizations of the well-known Banach contraction mapping principle in the literature. In particular, Matthews [1, 2] introduced the notion of a partial metric space as part of the study of denotational semantics of dataflow networks, showing that the Banach contraction mapping theorem can be generalized to the partial metric context for applications in program verification. After that, fixed point results in partial metric spaces have been studied by many authors. For example, Matthews [2] proved a Banach fixed point theorem for a self-map $T$ of a partial metric spaces $(X, p)$ under the following contractive condition:

$$
p(T x, T y) \leq c p(x, y), \quad \forall x, y \in X,
$$

where $0 \leq c<1$. Altun and Erduran [3] improved (1) by the following contractive condition:

$$
p(T x, T y) \leq \varphi(p(x, y)), \quad \forall x, y \in X,
$$

where $\varphi: \mathbb{R}_{+} \rightarrow \mathbb{R}_{+}$is a continuous nondecreasing function such that $\varphi(t)<t$ for all $t>0$ and proved the existence of the unique fixed point. Altun et al. [4] and Altun and Sadarangani [5] improved (2) by the following contractive condition:

$$
\begin{aligned}
& p(T x, T y) \leq \varphi(\max \{p(x, y), p(x, T x), p(y, T y), \\
& \left.\left.\quad \frac{1}{2}[p(x, T y)+p(y, T x)]\right\}\right),
\end{aligned}
$$

where $\varphi: \mathbb{R}_{+} \rightarrow \mathbb{R}_{+}$is continuous nondecreasing function such that $\varphi(t)<t$ and the series $\sum_{n \geq 1} \varphi^{n}(t)$ converges for all $t>0$ and proved the existence of the unique fixed point. Romaguera [6] improved (3) by assuming that $\varphi: \mathbb{R}_{+} \rightarrow \mathbb{R}_{+}$ is a right upper semicontinuous function such that $\varphi(t)<t$ for all $t>0$ and proved the existence of the unique fixed point without assuming that the series $\sum_{n \geq 1} \varphi^{n}(t)$ converges for all $t>0$. Abdeljawad et al. [7] proved the existence of fixed point for self-maps $T$ of $(X, p)$ under the following:

$$
p(T x, T y) \leq \psi(p(x, y))-\phi(p(x, y)), \quad \forall x, y \in X,
$$

where $\psi, \phi: \mathbb{R}_{+} \rightarrow \mathbb{R}_{+}$are continuous nondecreasing function such that $\varphi(t)=\psi(t)=0$ if and only if $t=0$. Abdeljawad et al. [8] proved the existence of common fixed point for selfmaps $S$ and $T$ of $(X, p)$ under the following:

$$
p(T x, S y) \leq N(x, y)-\phi(N(x, y)), \quad \forall x, y \in X,
$$

where $N(x, y)=\max \{p(x, y), p(T x, x), p(y, S y),(1 / 2)[p(T x$, $y)+p(x, S y)]\}, \phi: \mathbb{R}_{+} \rightarrow \mathbb{R}_{+}$is a continuous, nondecreasing function with $\phi(t)>0$ for all $t>0$ and $\phi(0)=0$.

Recently, Haghi et al. made [9] a paper which stated that we should "be careful on partial metric fixed point results" along with giving some results. The authors noted that some fixed point theorems in partial metric spaces can be obtained from the corresponding results in metric spaces. Abdeljawad et al. [10] used the technique of Haghi et al. in [9] to partial $b$-metric spaces. Reminded of the warning of Haghi et al. [9], one can see that the main characteristic of a partial metric 
space is that the self-distance $p(x, x)$ of a point $x$ may be not zero. But the all above contraction conditions are not proper for the characteristics. For example, if $(X, p)$ is a partial metric space and satisfies $p_{0}=\inf \{p(x, x): x \in X\}>0$, then the all above contraction conditions do not hold at any fixed point in this partial metric space $(X, p)$. This problem also shows that the abovementioned contractive conditions are only metric type contractive conditions but do not reflect the structure of a partial metric space. On the other hand, Ilić et al. [11] proved a more reasonable contraction principle in the partial metric space in which they used self-distance terms. They gave the following linear contractive condition allowing use of selfdistance in the contractive condition so that completeness, rather than the 0 -completeness, of the partial metric space is needed:

$$
\begin{aligned}
& p(T x, T y) \leq \max \{\alpha p(x, y), p(x, x), p(y, y)\} \\
& \forall x, y \in X,
\end{aligned}
$$

where $\alpha \in[0,1)$. After that, Chi et al. [12] gave the following linear generalization of (6):

$$
\begin{gathered}
p(T x, T y) \leq \max \{a p(x, y), b p(x, T x), c p(y, T y), \\
d[p(x T y)+p(y, T x)], p(x, x), p(y, y)\},
\end{gathered}
$$

$\forall x, y \in X$, where $a, b, c \in[0,1)$ and $d \in[0,1 / 2)$. Abdeljawad and Zaidan [13] gave the following linear generalization of (6):

$$
p(T x, T y) \leq \max \{\varphi(p(x, y)), p(x, x), p(y, y)\},
$$

where $\varphi:[0, \infty) \rightarrow[0, \infty)$ is an increasing function such that $f(t)=t-\varphi(t)$ is increasing with $f^{-1}$ being right continuous at 0 . Also assume $\lim _{n \rightarrow \infty} \varphi^{n}(t)=0$ for all $t \geq 0$ (and hence $\varphi(0)=0$ and $\varphi(t)<t$ for $t>0)$. The method of self-distance term is also used in [14-16]. In [17], the authors formulated the concept of $M$-metric spaces as a generalization of partial metric spaces, as well as a generalization of metric spaces. It is worth mentioning that the technique of Haghi et al. in [9] also is not applicable in $M$-metric spaces and the contraction condition presented in this paper is also not reflecting the structure of a $M$-metric space. After that, very recently, Abodayeh et al. [18] succeeded in studying $M$-metric and partial metric spaces topologically and proved a Caristi type fixed point theorem there by using self-distance term in their contractive conditions. For more general fixed point theorems, one can refer to $[19,20]$. In [19], the authors proved some common fixed point theorems for four mappings in partial metric spaces by using weakly compatible concept. In [20], the authors proved some fixed point theorems in more general ordered partial $b$-metric spaces. But these results still have similar gap between the contractive conditions and the structure of the spaces.

Inspired by the above works, in the present manuscript, we will focus on presenting a new nonlinear contraction principle on partial metric spaces for two mappings by using self-distance term in contractive conditions and proving the existence of common fixed point. Our result generalize and improve the results in $[2-8,11-14]$. We also give some examples to show our results and apply our results to study the existence of common bounded solution of the system of functional equations arising in dynamic programing.

\section{Preliminaries}

A partial metric space (see, e.g., [1]) is a pair $(X, p: X \times$ $X \rightarrow \mathbb{R}_{+}$) (where $\mathbb{R}_{+}$denotes the set of all nonnegative real numbers) such that for all $x, y, z \in X$

(PM1) $p(x, y)=p(y, x)$;

(PM2) $x=y$ if and only if $p(x, y)=p(x, x)=p(y, y)$;

(PM3) $p(x, x) \leq p(x, y)$;

(PM4) $p(x, z) \leq p(x, y)+p(y, z)-p(y, y)$.

Each partial metric $p$ on $X$ generates a $T_{0}$ topology $\tau_{p}$ on $X$ with a base of the family of open $p$-balls $\left\{B_{p}(x, \varepsilon): x \in\right.$ $X, \varepsilon>0\}$, where $B_{p}(x, \varepsilon)=\{y \in X: p(x, y)<p(x, x)+\varepsilon\}$ for all $x \in X$ and $\varepsilon>0$.

In a partial metric space, the concepts of convergence, the Cauchy sequence, completeness, and continuity are defined as follows.

Definition 1 (see $[1,2])$. (i) A sequence $\left\{x_{n}\right\}$ in a partial metric space $(X, p)$ converges to $x \in X$ if and only if $p(x, x)=$ $\lim _{n \rightarrow \infty} p\left(x, x_{n}\right)$.

(ii) A sequence $\left\{x_{n}\right\}$ in a partial metric space $(X, p)$ is called Cauchy if $\lim _{n, m \rightarrow \infty} p\left(x_{n}, x_{m}\right)$ exists and is finite.

(iii) A partial metric space $(X, p)$ is said to be complete if every Cauchy sequence $\left\{x_{n}\right\}$ in $X$ converges, with respect to $\tau_{p}$, to a point $x \in X$ such that $p(x, x)=\lim _{n, m \rightarrow \infty} p\left(x_{n}, x_{m}\right)$.

Example 2. Let $X=\mathbb{R}_{+}$and define $p(x, y)=\max \{x, y\}$, for all $x, y \in X$. Then $(X, p)$ is a complete partial metric space. It is clear that $p$ is not a (usual) metric.

\section{Main Results}

Let $(X, p)$ be a partial metric space, $A, B$ be self-maps of a set $X$, and

$$
\begin{aligned}
& M(x, y)=\max \{p(x, y), p(x, A x), p(y, B y), \\
& \left.\frac{[p(x, B y)+p(y, A x)]}{2}\right\},
\end{aligned}
$$

for any $x, y \in X$. For any $x_{0} \in X$, the sequence $\left\{x_{n}\right\}$ defined by $x_{2 n-1}=A x_{2 n-2}$ and $x_{2 n}=B x_{2 n-1}$, for $n \in N$, is called an $A, B$-iteration sequence of $x_{0}$ We first present the following contraction condition.

(C1) $p(A x, B y) \leq \max \{\phi(M(x, y)), p(x, x), p(y, y)\}$, for any $x, y \in X$, where $\phi:[0, \infty) \rightarrow[0, \infty)$ is uppersemicontinuous (i.e., $\lim \sup _{t \rightarrow t_{0}} \phi(t) \leq \phi\left(t_{0}\right), \forall t_{0} \in[0, \infty)$ ), such that $\phi(t)<t$ for any $t>0$.

Theorem 3. Let $A, B$ be self-mappings of a complete partial metric space $(X, p)$ such that (C1) holds; then $A, B$ have a common fixed point $z \in X$. 
Proof. Fix $x \in X$. Let $\left\{x_{n}\right\}$ be $A, B$-iteration sequence of $x_{0}=$ $x$. For $n \in \mathbf{N}$ define $p_{n}=p\left(x_{n}, x_{n+1}\right)$.

Step one: we prove that $\left\{p_{n}\right\}$ is a decreasing sequence; that is, for any $k \in \mathbf{N}$,

$$
p_{k+1} \leq p_{k} .
$$

(1) Assume that $k=2 n$. Then $p_{2 n+1}=p\left(x_{2 n+1}, x_{2 n+2}\right)=$ $p\left(A x_{2 n}, B x_{2 n+1}\right)$, and

$$
\begin{aligned}
M & \left(x_{2 n}, x_{2 n+1}\right)=\max \left\{p\left(x_{2 n}, x_{2 n+1}\right), p\left(x_{2 n}, A x_{2 n}\right),\right. \\
& p\left(x_{2 n+1}, B x_{2 n+1}\right), \\
& \left.\frac{1}{2}\left[p\left(x_{2 n}, B x_{2 n+1}\right)+p\left(x_{2 n+1}, A x_{2 n}\right)\right]\right\} \\
& =\max \left\{p\left(x_{2 n}, x_{2 n+1}\right), p\left(x_{2 n}, x_{2 n+1}\right),\right. \\
& p\left(x_{2 n+1}, x_{2 n+2}\right), \\
& \left.\frac{1}{2}\left[p\left(x_{2 n}, x_{2 n+2}\right)+p\left(x_{2 n+1}, x_{2 n+1}\right)\right]\right\} \\
& =\max \left\{p\left(x_{2 n}, x_{2 n+1}\right), p\left(x_{2 n+1}, x_{2 n+2}\right)\right\} \\
& =\max \left\{p_{2 n}, p_{2 n+1}\right\} .
\end{aligned}
$$

It follows from $(\mathrm{C} 1)$ that

$$
\begin{aligned}
& p_{2 n+1}=p\left(x_{2 n+1}, x_{2 n+2}\right)=p\left(A x_{2 n}, B x_{2 n+1}\right) \\
& \quad \leq \max \left\{\phi\left(M\left(x_{2 n}, x_{2 n+1}\right)\right), p\left(x_{2 n}, x_{2 n}\right),\right. \\
& \left.p\left(x_{2 n+1}, x_{2 n+1}\right)\right\}=\max \left\{\phi\left(\max \left\{p_{2 n}, p_{2 n+1}\right\}\right),\right. \\
& \left.p\left(x_{2 n}, x_{2 n}\right), p\left(x_{2 n+1}, x_{2 n+1}\right)\right\} .
\end{aligned}
$$

If $\phi\left(\max \left\{p_{2 n}, p_{2 n+1}\right\}\right)>\max \left\{p\left(x_{2 n}, x_{2 n}\right), p\left(x_{2 n+1}, x_{2 n+1}\right)\right\}$, we have that

$$
\begin{gathered}
p_{2 n+1} \leq \max \left\{\phi\left(\max \left\{p_{2 n}, p_{2 n+1}\right\}\right), p\left(x_{2 n}, x_{2 n}\right),\right. \\
\left.p\left(x_{2 n+1}, x_{2 n+1}\right)\right\}=\phi\left(\max \left\{p_{2 n}, p_{2 n+1}\right\}\right) .
\end{gathered}
$$

This implies that $p_{2 n+1}<p_{2 n}$. If $\phi\left(\max \left\{p_{2 n}, p_{2 n+1}\right\}\right) \leq$ $\max \left\{p\left(x_{2 n}, x_{2 n}\right), p\left(x_{2 n+1}, x_{2 n+1}\right)\right\}$, we have that

$$
p_{2 n+1} \leq \max \left\{p\left(x_{2 n}, x_{2 n}\right), p\left(x_{2 n+1}, x_{2 n+1}\right)\right\} \leq p_{2 n} .
$$

Thus, we have proved that $p_{2 n+1} \leq p_{2 n}$.

(2) Assume that $k=2 n+1$; using a similar argument as in (1), one can verify that $p_{2 n+2} \leq p_{2 n+1}$.

Combining the results of (1) and (2), we may conclude that (10) holds. Hence there exists $r_{x} \geq 0$, such that $\lim _{n \rightarrow \infty} p_{n}=r_{x}$.

Step two: we prove that $\lim _{n \rightarrow \infty} p\left(x_{n}, x_{n}\right)=r_{x}$. Assume that $\varlimsup_{n \rightarrow \infty} p\left(x_{n}, x_{n}\right)=p_{x}$. Note that $p\left(x_{n}, x_{n}\right) \leq p_{n+1}$, and we can obtain that

$$
\varlimsup_{n \rightarrow \infty} p\left(x_{n}, x_{n}\right)=p_{x} \leq \lim _{n \rightarrow \infty} p_{n}=r_{x} .
$$

Since $\left\{p_{n}\right\}$ is decreasing, by the proof of Step one, we have that

$$
p_{n+1} \leq \max \left\{\phi\left(p_{n}\right), p\left(x_{n}, x_{n}\right), p\left(x_{n+1}, x_{n+1}\right)\right\} .
$$

If there are infinite number of $n$, such that

$$
\max \left\{\phi\left(p_{n}\right), p\left(x_{n}, x_{n}\right), p\left(x_{n+1}, x_{n+1}\right)\right\}=\phi\left(p_{n}\right),
$$

then we have that $p_{n+1} \leq \phi\left(p_{n}\right)$. Thus, by letting $n \rightarrow \infty$ we can obtain that $r_{x} \leq \phi\left(r_{x}\right)$. This implies that $r_{x}=0$, so we have that $p_{x}=0$. Thus,

$$
\lim _{n \rightarrow \infty} p\left(x_{n}, x_{n}\right)=r_{x}=p_{x}=0 .
$$

Therefore, if $r_{x}>0$, then there exists $K>0$, such that, for any $n>K$,

$$
\phi\left(p_{n}\right)<\max \left\{p\left(x_{n}, x_{n}\right), p\left(x_{n+1}, x_{n+1}\right)\right\},
$$

so we have that

$$
p_{n+1} \leq \max \left\{p\left(x_{n}, x_{n}\right), p\left(x_{n+1}, x_{n+1}\right)\right\} .
$$

This has two cases. Case one: there exists $K_{1}>K$, such that $p_{n+1} \leq p\left(x_{n}, x_{n}\right)$, for any $n>K_{1}$, or $p_{n+1} \leq p\left(x_{n+1}, x_{n+1}\right)$, for any $n>K_{1}$. In this case we have that

$$
\begin{aligned}
\lim _{n \rightarrow \infty} p_{n+1} & =r_{x} \leq \varliminf_{n \rightarrow \infty} p\left(x_{n}, x_{n}\right) \leq \varlimsup_{n \rightarrow \infty} p\left(x_{n}, x_{n}\right) \\
& \leq \lim _{n \rightarrow \infty} p_{n}=r_{x},
\end{aligned}
$$

and, thus, $\lim _{n \rightarrow \infty} p\left(x_{n}, x_{n}\right)=r_{x}$. Case two: if case one does not hold, then there are subsequences $\left\{n_{k}\right\},\left\{n_{k}^{\prime}\right\}$, such that $\{n\}=\left\{n_{k}\right\} \cup\left\{n_{k}^{\prime}\right\}, n>K$, and

$$
\begin{aligned}
& p_{n_{k}+1} \leq p\left(x_{n_{k}}, x_{n_{k}}\right), \\
& p_{n_{k}^{\prime}+1} \leq p\left(x_{n_{k}^{\prime}+1}, x_{n_{k}^{\prime}+1}\right) .
\end{aligned}
$$

In this case we also have that

$$
\begin{aligned}
\lim _{k \rightarrow \infty} p\left(x_{n_{k}}, x_{n_{k}}\right) & =r_{x}, \\
\lim _{k \rightarrow \infty} p\left(x_{n_{k}^{\prime}+1}, x_{n_{k}^{\prime}+1}\right) & =r_{x} .
\end{aligned}
$$

From this, we obtain that $\lim _{n \rightarrow \infty} p\left(x_{n}, x_{n}\right)=r_{x}$. By the conclusion of Step one, we know that $p_{n+1} \geq r_{x}$ for any $n \in N$. Then, by case one and case two, we obtain that there exists positive integer $L>0$, such that $p\left(x_{n}, x_{n}\right) \geq r_{x}$ for any $n>L$.

Step three: we prove that

$$
\lim _{n, m \rightarrow \infty} p\left(x_{n}, x_{m}\right)=r_{x}
$$

By the conclusion of Step two, we get that $p\left(x_{n}, x_{m}\right) \geq$ $p\left(x_{n}, x_{n}\right) \geq r_{x}$ for any $n>L$. Thus, if (24) does not hold, then there exist $\varepsilon_{0}>0$ and increasing sequence of integers $\{m(k)\}$ and $\{n(k)\}$ such that, for all integers $k$,

$$
\begin{aligned}
n(k) & >m(k)>k+2, \\
p\left(x_{n(k)}, x_{m(k)}\right) & \geq r_{x}+\varepsilon_{0} .
\end{aligned}
$$


Since $\lim _{n \rightarrow \infty} p\left(x_{n}, x_{n}\right)=\lim _{n \rightarrow \infty} p\left(x_{n+1}, x_{n}\right)=r_{x}$, we have that

$$
\begin{gathered}
p\left(x_{m(k)}, x_{m(k)}\right)<r_{x}+\varepsilon_{0}, \\
p\left(x_{m(k)+1}, x_{m(k)}\right)<r_{x}+\varepsilon_{0},
\end{gathered}
$$

when $m(k)$ is large enough. So we can assume that $n(k)$ is the minimum positive integer such that (25) holds. Then we have that

$$
p\left(x_{n(k)-1}, x_{m(k)}\right)<r_{x}+\varepsilon_{0} .
$$

Using (25), (27), and the triangle inequality for partial metric we have

$$
\begin{aligned}
r_{x}+\varepsilon_{0} \leq & p\left(x_{n(k)}, x_{m(k)}\right) \\
\leq & p\left(x_{n(k)}, x_{n(k)-1}\right)+p\left(x_{n(k)-1}, x_{m(k)}\right) \\
& -p\left(x_{n(k)-1}, x_{n(k)-1}\right) \\
\leq & p\left(x_{n(k)}, x_{n(k)-1}\right)+r_{x}+\varepsilon_{0} \\
& -p\left(x_{n(k)-1}, x_{n(k)-1}\right) .
\end{aligned}
$$

Thus,

$$
\begin{array}{r}
\lim _{k \rightarrow \infty} p\left(x_{n(k)}, x_{m(k)}\right)=r_{x}+\varepsilon_{0}, \\
\lim _{k \rightarrow \infty} p\left(x_{n(k)-1}, x_{m(k)}\right)=r_{x}+\varepsilon_{0} .
\end{array}
$$

Again, the triangle inequality gives us

$$
\begin{aligned}
p\left(x_{n(k)}, x_{m(k)-1}\right) \leq & p\left(x_{n(k)}, x_{m(k)}\right) \\
& +p\left(x_{m(k)}, x_{m(k)-1}\right) \\
& -p\left(x_{m(k)}, x_{m(k)}\right), \\
p\left(x_{n(k)}, x_{m(k)}\right) \leq & p\left(x_{n(k)}, x_{m(k)-1}\right) \\
& +p\left(x_{m(k)-1}, x_{m(k)}\right) \\
& -p\left(x_{m(k)-1}, x_{m(k)-1}\right) .
\end{aligned}
$$

Thus,

$$
\begin{aligned}
& p\left(x_{n(k)}, x_{m(k)}\right)-p\left(x_{m(k)-1}, x_{m(k)}\right) \\
& \quad+p\left(x_{m(k)-1}, x_{m(k)-1}\right) \leq p\left(x_{n(k)}, x_{m(k)-1}\right) \\
& \quad \leq p\left(x_{n(k)}, x_{m(k)}\right)+p\left(x_{m(k)}, x_{m(k)-1}\right) \\
& \quad-p\left(x_{m(k)}, x_{m(k)}\right) .
\end{aligned}
$$

Letting $k \rightarrow \infty$, we get

$$
\lim _{k \rightarrow \infty} p\left(x_{n(k)}, x_{m(k)-1}\right)=r_{x}+\varepsilon_{0} .
$$

Similarly, we can prove that

$$
\begin{aligned}
& \lim _{k \rightarrow \infty} p\left(x_{n(k)}, x_{m(k)+1}\right)=\lim _{k \rightarrow \infty} p\left(x_{n(k)-1}, x_{m(k)+1}\right) \\
& =\lim _{k \rightarrow \infty} p\left(x_{n(k)-1}, x_{m(k)-1}\right)=r_{x}+\varepsilon_{0}, \\
& \lim _{k \rightarrow \infty} p\left(x_{n(k)+1}, x_{m(k)+1}\right)=\lim _{k \rightarrow \infty} p\left(x_{n(k)+1}, x_{m(k)}\right) \\
& =\lim _{k \rightarrow \infty} p\left(x_{n(k)+1}, x_{m(k)-1}\right)=r_{x}+\varepsilon_{0},
\end{aligned}
$$

putting

$$
\begin{aligned}
& \left(x_{n(k)}^{*}, x_{m(k)}^{*}\right) \\
& = \begin{cases}\left(x_{n(k)-1}, x_{m(k)-1}\right), & n(k) \text { is odd, } m(k) \text { is even, } \\
\left(x_{n(k)-1}, x_{m(k)}\right), & n(k) \text { is odd, } m(k) \text { is odd, } \\
\left(x_{n(k)}, x_{m(k)-1}\right), & n(k) \text { is even, } m(k) \text { is even, } \\
\left(x_{n(k)}, x_{m(k)}\right), & n(k) \text { is even, } m(k) \text { is odd. }\end{cases}
\end{aligned}
$$

By use of (29), (32), and (33), we have

$$
\begin{aligned}
& \lim _{k \rightarrow \infty} M\left(x_{n(k)}^{*}, x_{m(k)}^{*}\right)=\lim _{k \rightarrow \infty} \max \left\{p\left(x_{n(k)}^{*}, x_{m(k)}^{*}\right),\right. \\
& p\left(x_{n(k)}^{*}, A x_{n(k)}^{*}\right), p\left(x_{m(k)}^{*}, B x_{m(k)}^{*}\right), \\
& \left.\frac{\left[p\left(x_{n(k)}^{*}, B x_{m(k)}^{*}\right)+p\left(A x_{n(k)}^{*}, x_{m(k)}^{*}\right)\right]}{2}\right\}=r_{x}+\varepsilon_{0} .
\end{aligned}
$$

And $\lim _{k \rightarrow \infty} p\left(A x_{n(k)}^{*}, B x_{m(k)}^{*}\right)=r_{x}+\varepsilon_{0}$. But, by using $(\mathrm{C} 1)$, we know that

$$
\begin{gathered}
p\left(A x_{n(k)}^{*}, B x_{m(k)}^{*}\right) \leq \max \left\{\phi\left(M\left(x_{n(k)}^{*}, x_{m(k)}^{*}\right)\right),\right. \\
\left.p\left(x_{n(k)}^{*}, x_{n(k)}^{*}\right), p\left(x_{m(k)}^{*}, x_{m(k)}^{*}\right)\right\} .
\end{gathered}
$$

Thus, by letting $k \rightarrow \infty$, we get

$$
r_{x}+\varepsilon_{0} \leq \max \left\{\phi\left(r_{x}+\varepsilon_{0}\right), r_{x}, r_{x}\right\}<r_{x}+\varepsilon_{0} .
$$

This yields a contradiction. Therefore, $\left\{x_{n}\right\}$ is a Cauchy sequence. By the completeness of $(X, p),\left\{x_{n}\right\}$ is a convergent sequence. Then there exists $\widetilde{x} \in X$ such that

$$
\begin{aligned}
\lim _{n \rightarrow \infty} p\left(x_{n}, \tilde{x}\right) & =\lim _{n, m \rightarrow \infty} p\left(x_{n}, x_{m}\right)=\lim _{n \rightarrow \infty} p\left(x_{n}, x_{n}\right) \\
& =r_{x}=p(\tilde{x}, \tilde{x}) .
\end{aligned}
$$

Step four: we prove that

$$
p(\tilde{x}, A \tilde{x})=p(\tilde{x}, B \tilde{x})=p(\tilde{x}, \tilde{x}) .
$$

For any $u \in X$, we have that

$$
\begin{aligned}
& p(u, \tilde{x}) \leq p\left(u, x_{n}\right)+p\left(x_{n}, \tilde{x}\right)-p\left(x_{n}, x_{n}\right), \\
& p\left(u, x_{n}\right) \leq p(u, \tilde{x})+p\left(\tilde{x}, x_{n}\right)-p(\tilde{x}, \tilde{x}) .
\end{aligned}
$$


This implies that

$$
\lim _{n \rightarrow \infty} p\left(u, x_{n}\right)=p(u, \tilde{x})
$$

Again note that

$$
\lim _{n \rightarrow \infty} x_{2 n}=\lim _{n \rightarrow \infty} B x_{2 n-1}=\tilde{x},
$$

and we obtain that

$$
\begin{aligned}
& p(\tilde{x}, A \tilde{x})=\lim _{n \rightarrow \infty} p\left(A \tilde{x}, B x_{2 n-1}\right) \\
& \quad \leq \lim _{n \rightarrow \infty} \max \left\{\phi\left(M\left(\tilde{x}, x_{2 n-1}\right)\right), p(\tilde{x}, \tilde{x}),\right. \\
& \left.\quad p\left(x_{2 n-1}, x_{2 n-1}\right)\right\}=\max \{\phi(p(\tilde{x}, A \tilde{x})), p(\tilde{x}, \tilde{x})\} .
\end{aligned}
$$

$$
\begin{aligned}
& p\left(x_{n}^{k}, x_{n}^{k}\right) \leq p\left(x_{n}^{k}, x_{n+1}^{k}\right) \leq \cdots \leq p\left(x_{1}^{k}, x_{2}^{k}\right)=p\left(A x_{k}, B A x_{k}\right) \\
& \leq \max \left\{\phi\left(\max \left\{p\left(x_{k}, A x_{k}\right), p\left(x_{k}, A x_{k}\right), p\left(A x_{k}, B A x_{k}\right), \frac{1}{2}\left[p\left(x_{k}, B A x_{k}\right)+p\left(A x_{k}, A x_{k}\right)\right]\right\}\right), p\left(x_{k}, x_{k}\right), p\left(A x_{k}, A x_{k}\right)\right\} \\
& =\max \left\{\phi\left(\max \left\{p\left(x_{k}, x_{k}\right), p\left(A x_{k}, B A x_{k}\right)\right\}\right), p\left(x_{k}, x_{k}\right)\right\} \leq p\left(x_{k}, x_{k}\right) .
\end{aligned}
$$

This shows that, by using the conclusion of Step two,

This implies that

$$
p\left(\widetilde{x_{k}}, \widetilde{x_{k}}\right)=\lim _{n \rightarrow \infty} p\left(x_{n}^{k}, x_{n}^{k}\right) \leq p\left(x_{k}, x_{k}\right) .
$$

Thus, we have that

$$
l_{p} \leq p\left(\widetilde{x_{k}}, \widetilde{x_{k}}\right) \leq p\left(x_{k}, x_{k}\right)<l_{p}+\frac{1}{k} .
$$

and $l_{p}=\inf \{p(\tilde{x}, \tilde{x}): \tilde{x} \in \widetilde{X}\}$, we prove that there exists $\tilde{y} \in X$, such that $p(\tilde{y}, \tilde{y})=l_{p}$.

For any $k \in N$, we take $x_{k} \in \widetilde{X}$, such that

$$
p\left(x_{k}, x_{k}\right)<l_{p}+\frac{1}{k} \text {. }
$$

Assume that $\left\{x_{n}^{k}\right\}$ is the $A, B$-iterative sequence of $x_{k}$ and converges to $\widetilde{x_{k}}$. By using the conclusions of Step one and Step four, we obtain that

Since

$$
\begin{aligned}
& l_{p} \leq p\left(\widetilde{x_{k}}, \widetilde{x_{k}}\right) \leq p\left(\widetilde{x_{k}}, \widetilde{x}_{l}\right)=\lim _{n \rightarrow \infty} p\left(x_{n}^{k}, x_{n}^{l}\right)=\lim _{n \rightarrow \infty} p\left(A x_{2 n}^{k}, B x_{2 n-1}^{l}\right), \\
& p\left(A x_{2 n}^{k}, B x_{2 n-1}^{l}\right) \leq \max \left\{\phi\left(M\left(x_{2 n}^{k}, x_{2 n-1}^{l}\right)\right), p\left(x_{2 n}^{k}, x_{2 n}^{k}\right), p\left(x_{2 n-1}^{l}, x_{2 n-1}^{l}\right)\right\} \\
& =\max \left\{\phi\left(\max \left\{p\left(x_{2 n}^{k}, x_{2 n-1}^{l}\right), p\left(x_{2 n}^{k}, A x_{2 n}^{k}\right), p\left(x_{2 n-1}^{l}, B x_{2 n-1}^{l}\right), \frac{1}{2}\left[p\left(x_{2 n}^{k}, B x_{2 n-1}^{l}\right)+p\left(x_{2 n-1}^{l}, A x_{2 n}^{k}\right)\right]\right\}\right), p\left(x_{2 n}^{k}, x_{2 n}^{k}\right), p\left(x_{2 n-1}^{l}, x_{2 n-1}^{l}\right)\right\},
\end{aligned}
$$

we obtain that

$$
\begin{aligned}
l_{p} & \leq p\left(\widetilde{x_{k}}, \widetilde{x}_{l}\right)=\lim _{n \rightarrow \infty} p\left(x_{n}^{k}, x_{n}^{l}\right)=\lim _{n \rightarrow \infty} p\left(A x_{2 n}^{k},\right. \\
& \left.B x_{2 n-1}^{l}\right) \leq \varlimsup_{n \rightarrow \infty} \max \left\{\phi\left(M\left(x_{2 n}^{k}, x_{2 n-1}^{l}\right)\right),\right. \\
& \left.p\left(x_{2 n}^{k}, x_{2 n}^{k}\right), p\left(x_{2 n-1}^{l}, x_{2 n-1}^{l}\right)\right\} \\
& \leq \max \left\{\phi\left(p\left(\widetilde{x_{k}}, \widetilde{x}_{l}\right)\right), p\left(\widetilde{x_{k}}, \widetilde{x_{k}}\right), p\left(\widetilde{x_{l}}, \widetilde{x}_{l}\right)\right\} \\
& =\max \left\{p\left(\widetilde{x_{k}}, \widetilde{x_{k}}\right), p\left(\widetilde{x_{l}}, \widetilde{x}_{l}\right)\right\} \leq \max \left\{p\left(x_{k}, x_{k}\right),\right. \\
& \left.p\left(x_{l}, x_{l}\right)\right\}<l_{p}+\frac{1}{k}+\frac{1}{l} .
\end{aligned}
$$

This shows that $\lim _{k, l \rightarrow \infty} p\left(\widetilde{x_{k}}, \widetilde{x_{l}}\right)=l_{p}$; that is, $\left\{\widetilde{x_{n}}\right\}$ is a Cauchy sequence. Since the partial metric space $(X, p)$ is completed, there is $y \in X$, such that

$$
p(y, y)=\lim _{n \rightarrow \infty} p\left(\widetilde{x_{n}}, y\right)=\lim _{n, m \rightarrow \infty} p\left(\widetilde{x_{n}}, \widetilde{x_{m}}\right)=l_{p} .
$$

It follows from

$$
\begin{aligned}
& p\left(\widetilde{x_{n}}, A y\right) \leq p\left(\widetilde{x_{n}}, y\right)+p(y, A y)-p(y, y), \\
& p(y, A y) \leq p\left(y, \widetilde{x_{n}}\right)+p\left(\widetilde{x_{n}}, A y\right)-p\left(\widetilde{x_{n}}, \widetilde{x_{n}}\right)
\end{aligned}
$$

that

$$
\lim _{n \rightarrow \infty} p\left(\widetilde{x_{n}}, A y\right)=p(y, A y)
$$


On the other hand, we know that

$$
\begin{aligned}
p\left(\widetilde{x_{n}}, A y\right) & =\lim _{m \rightarrow \infty} p\left(A y, x_{2 m}^{n}\right)=\lim _{m \rightarrow \infty} p\left(A y, B x_{2 m-1}^{n}\right) \leq \varlimsup_{m \rightarrow \infty} \max \left\{\phi\left(M\left(y, x_{2 m-1}^{n}\right)\right), p(y, y), p\left(x_{2 m-1}^{n}, x_{2 m-1}^{n}\right)\right\} \\
& =\max \left\{\phi\left(\max \left\{p\left(y, \widetilde{x_{n}}\right), p(y, A y), p\left(\widetilde{x_{n}}, \widetilde{x_{n}}\right), \frac{1}{2}\left[p\left(y, \widetilde{x_{n}}\right)+p\left(\widetilde{x_{n}}, A y\right)\right]\right\}\right), p(y, y), p\left(\widetilde{x_{n}}, \widetilde{x_{n}}\right)\right\} .
\end{aligned}
$$

Then by letting $n \rightarrow \infty$ we get that

$$
\begin{aligned}
& p(y, A y)=\lim _{n \rightarrow \infty} p\left(\widetilde{x_{n}}, A y\right) \\
& \quad \leq \max \{\phi(\max \{p(y, y), p(y, A y)\}), p(y, y)\} .
\end{aligned}
$$

This implies that $p(y, A y) \leq p(y, y)$, so we have that

$$
p(y, A y)=p(y, y) \text {. }
$$

Similarly, one can prove that

$$
\begin{aligned}
\lim _{n \rightarrow \infty} p\left(\widetilde{x_{n}}, B y\right) & =p(y, B y), \\
p(y, B y) & =p(y, y) .
\end{aligned}
$$

Assume that $\left\{y_{n}\right\}$ is the $A, B$-iterative sequence of $y$ and converges to $\tilde{y}$. By using the conclusions of Step one and Step two, we get that

$$
\begin{aligned}
l_{p} & \leq p(\tilde{y}, \tilde{y}) \leq p\left(y_{n}, y_{n+1}\right) \leq \cdots \leq p(y, A y) \\
& =p(y, y)=l_{p},
\end{aligned}
$$

and, thus, $p(\tilde{y}, \tilde{y})=l_{p}$.

Step six: we prove that $A, B$ have unique common fixed point $u \in X$ with $p(u, u)=l_{p}$.

By using the conclusions of Step one, Step two, and Step five, we know that there is $K>0$, such that $p(\tilde{y}, \tilde{y}) \leq$ $p\left(y_{n}, y_{n}\right)$ for any $n>K$ and

$$
\begin{aligned}
l_{p} & =p(\widetilde{y}, \tilde{y}) \leq p\left(y_{n}, y_{n}\right) \leq p\left(y_{n}, y_{n+1}\right) \leq \cdots \\
& \leq p(y, A y)=p(y, y)=l_{p} .
\end{aligned}
$$

Thus, we have that

$$
\begin{aligned}
l_{p} & =p(\tilde{y}, \tilde{y})=p\left(y_{n}, y_{n}\right)=p\left(y_{n}, y_{n+1}\right)=\cdots \\
& =p(y, y), \quad n>K .
\end{aligned}
$$

It follows from (61) that for $n>K$

$$
\begin{aligned}
l_{p} & =p(\tilde{y}, \tilde{y})=p\left(y_{2 n}, y_{2 n}\right)=p\left(y_{2 n}, y_{2 n+1}\right) \\
& =p\left(y_{2 n+1}, y_{2 n+1}\right), \\
& =p\left(y_{2 n+1}, y_{2 n+2}\right)=p\left(y_{2 n+2}, y_{2 n+2}\right),
\end{aligned}
$$

and this shows that

$$
y_{2 n}=y_{2 n+1}=y_{2 n+2}
$$

that is

$$
y_{2 n}=y_{2 n+1}=A y_{2 n}=y_{2 n+2}=B y_{2 n+1}=B y_{2 n} .
$$

Then we have proved that $y_{2 n}$ is a common fixed point of $A$, $B$. Assume that $u, v \in X$ are two common fixed points of $A, B$, with the properties of $p(u, u)=p(v, v)=l_{p}$. Clearly, $u, v \in \widetilde{X}$, since the $A, B$-iterative sequence of a fixed point is a constant sequence. By using $(\mathrm{C} 1)$ we have that

$$
\begin{aligned}
p(u, v) & =p(A u, B v) \\
& \leq \max \{\phi(M(u, v)), p(u, u), p(v, v)\} \\
& =\max \left\{\phi(p(u, v)), l_{p}\right\},
\end{aligned}
$$

and this implies that $p(u, v)=p(u, u)=p(v, v)=l_{p}$. Thus, $u=v$. The proof is completed.

Remark 4. If $(X, p)$ is a metric space, then contraction condition $(\mathrm{C} 1)$ reduces to the corresponding contraction conditions on metric spaces. Thus, the corresponding results in the set of a metric space is a special case of our results. But, in a partial metric space, since the partial metric space topology is only $T_{0}$ topology, the limit of a sequence $\left\{x_{n}\right\}$ in a partial metric space $(X, p)$ is not unique (refer to Remark 7 in [18]), and this induced that the fixed point may not be unique. In Theorem 3, we can only get that $A, B$ have unique common fixed point $u \in\left\{y \in X: p(y, y)=l_{p}\right\}$.

Remark 5. In Theorem 3, if $A=B$, then the condition " $\phi$ : $[0, \infty) \rightarrow[0, \infty)$ is upper-semicontinuous" can be replaced by " $\phi:[0, \infty) \rightarrow[0, \infty)$ is upper-semicontinuous from the right." It is only needed to modify the proof of Theorem 3.

Remark 6. If $A=B$, the conclusion of Theorem 3 is a generalization and improvement for the results in [2-6, 11-13].

Remark 7. In Theorem 3, if $\phi$ is replaced by $\Phi=\psi-\phi$, where $\psi, \phi: \mathbb{R}_{+} \rightarrow \mathbb{R}_{+}$are continuous function such that $\phi(t)=$ $\psi(t)=0$ if and only if $t=0$, then the conclusion of Theorem 3 in this case is a generalization and improvement for the results in $[7,8]$.

Remark 8. Let $\left(X, S_{p}\right)$ be a complete partial $S$-metric space and $T$ be a self-mapping on $X$ (see [14]), if we define $p(x, y)=$ $S_{p}(x, x, y), \forall x, y \in X$, then $(X, p)$ is a complete partial metric space, and the contraction condition

$$
\begin{aligned}
& S_{p}(T x, T x, T y) \\
& \quad \leq \max \left\{k S_{p}(x, x, y), S_{p}(x, x, x), S_{p}(y, y, y)\right\}
\end{aligned}
$$


of Theorem 2.1 in [14] will become

$$
p(T x, T y) \leq \max \{k p(x, y), p(x, x), p(y, y)\} .
$$

The contraction condition

$$
\begin{aligned}
& S_{p}(T x, T x, T y) \\
& \quad \leq \max \left\{k S_{p}(x, x, y), \frac{S_{p}(x, x, x)+S_{p}(y, y, y)}{2}\right\}
\end{aligned}
$$

of Theorem 2.2 in [14] will become

$$
p(T x, T y) \leq \max \left\{k p(x, y), \frac{p(x, x)+p(y, y)}{2}\right\} .
$$

Therefore, our Theorem 3 is a generalization and improvement for the results in [14].

Remark 9. Some nice results for more general fixed point theorems were proved in $[19,20]$. In [19], the authors proved some common fixed point theorems for four mappings in partial metric spaces by using weakly compatible concept and some control functions, but these control functions are different from upper-semicontinuous ones. In [20], the authors proved some fixed point theorems in more general ordered partial $b$-metric spaces. However, there is still the problem mentioned in the Introducion. That is, the contractive conditions presented in these papers do not reflect the structure of the partial metric space or partrial $G_{p b}$ metric space. Thus, our results can not be deduced from the results in $[19,20]$. But the results in $[19,20]$ also show that there is still more greater research space to prove some contraction principles with the contractive conditions includeing selfdistance terms.

The following simple example is an illustration of our extension.

Example 10. Suppose that $X=\mathbb{R}_{+}$and $p(x, y)=e^{\max \{x, y\}}$. Then $(X, p)$ is a partial metric space. Suppose that $A: X \rightarrow X$ such that

$$
A x= \begin{cases}\frac{x^{2}}{1+x}, & x \neq 1, \\ 1, & x=1\end{cases}
$$

for all $x \in X$ and $\phi:[0, \infty) \rightarrow[0, \infty)$ and $\phi(t)=t /\left(1+t^{2}\right)$, for any $x, y \in \mathbb{R}_{+}$. Then, for all $x, y \in X$ with $x \geq y$, we have that

$$
\begin{aligned}
& p(A x, A y)=e^{\max \left\{x^{2} /(1+x), y^{2} /(1+y)\right\}}=e^{x^{2} /(1+x)}, \\
& M(x, y)=\max \left\{e^{x}, e^{x}, e^{y}, \frac{1}{2}\left[e^{x}+e^{\max \left\{y, x^{2} /(1+x)\right\}}\right]\right\} \\
& \quad=e^{x}, \\
& p(A x, A y)=e^{x^{2} /(1+x)} \leq \max \left\{\phi\left(e^{x}\right), e^{x}, e^{y}\right\} .
\end{aligned}
$$

Therefore, the conditions of Theorem 3 are satisfied. Indeed $x=0$ and $x=1$ are the fixed point. But

$$
\begin{aligned}
p(A x, A y) & =e^{x^{2} /(1+x)}>\phi(M(x, y))=\phi\left(e^{x}\right) \\
& =\frac{e^{x}}{1+e^{2 x}} .
\end{aligned}
$$

Thus, we cannot apply the results of [2-8] to this example.

Example 11. Suppose that $X=\mathbb{R}^{+}$and $p(x, y)=\max \{x, y\}$. Then $(X, p)$ is a partial metric space. Define $A, B: X \rightarrow X$ by $A x=x / 2(1+x)$ and $B x=x-x^{2} / 2(1+x)$ for all $x \in X$ and define $\phi:[0, \infty) \rightarrow[0, \infty)$ by $\phi(t)=\ln (1+t)$, for all $t \in[0,1]$. Then, for all $x, y \in X$, we have

$$
\begin{gathered}
p(A x, B y)=\max \left\{\frac{x}{2(1+x)}, y-\frac{y^{2}}{2(1+y)}\right\}, \\
M(x, y)=\max \{p(x, y), p(x, A x), p(y, B y), \\
\left.\frac{[p(x, B y)+p(y, A x)]}{2}\right\}=\max \{x, y\} .
\end{gathered}
$$

If $x \geq y$, then $p(A x, B y)=x / 2(1+x) \leq \phi(x)=\phi(M(x, y))$. If $y>x$ and $x / 2(1+x) \leq y-y^{2} / 2(1+y)$, we have that

$$
\begin{aligned}
p(A x, B y) & =y-\frac{y^{2}}{2(1+y)}>\ln (1+y)=\phi(y) \\
& =\phi(M(x, y)) .
\end{aligned}
$$

Thus, we cannot apply the results of [2-8] to this example. But in both cases $x \geq y$ and $y>x$, and we always have that

$$
p(A x, B y) \leq \max \{\phi(M(x, y)), p(x, x), p(y, y)\}
$$

holds. Thus, the conditions of Theorem 3 are satisfied and $A$, $B$ have a common fixed point $x=0$.

\section{An Application to a Dynamical Process}

Generally, a dynamical process consists of a state space and a decision space. The state space is the set of the initial state, actions, and transition model of the process; the decision space is the set of possible actions that are allowed for the process.

In this section we assume that $X$ and $Y$ are Banach spaces; $X_{1} \subset X$ is a state space and $Y_{1} \subset Y$ is a decision space. It is well-known that the dynamic programing provides useful tools for mathematical optimization and computer programing as well. In particular, the problem of dynamic 
programing related to multistage process reduces to the problem of solving the system of functional equations

$$
\begin{array}{r}
q_{1}(x)=\sup _{y \in Y_{1}}\left\{f(x, y)+G\left(x, y, q_{1}\left(\tau_{1}(x, y)\right)\right)\right\}, \\
x \in X_{1}, \\
q_{2}(x)=\sup _{y \in Y_{1}}\left\{f(x, y)+H\left(x, y, q_{2}\left(\tau_{2}(x, y)\right)\right)\right\}, \\
x \in X_{1},
\end{array}
$$

where $x$ and $y$ signify the state and decision vectors, $f: X_{1} \times$ $Y_{1} \rightarrow \mathbb{R}, G, H: X_{1} \times Y_{1} \times \mathbb{R} \rightarrow \mathbb{R}$, and $\tau_{i}: X_{1} \times Y_{1} \rightarrow X_{1}$ $(i=1,2)$ represent the transformations of the process, and $q_{1}$, $q_{2}$ denote the optimal return functions with the initial state $x$. However, for the detailed background of the problem, the reader can refer to $[21,22]$. Here, we study the existence of the common bounded solution of the system of functional equations (76).

Let $Z$ denote the set of all bounded real-valued functions on $X_{1}$ and, for an arbitrary $z \in Z$, define $\|z\|=\sup _{x \in X_{1}}|z(x)|$. Clearly, $(Z,\|\cdot\|)$ endowed with the metric $d$ defined by

$$
d\left(z_{1}, z_{2}\right)=\sup _{x \in X_{1}}\left|z_{1}(x)-z_{2}(x)\right|, \quad z_{1}, z_{2} \in Z
$$

is a Banach space. Indeed, the convergence in the space $Z$ with respect to $\|\cdot\|$ is uniform. Thus, if we consider a Cauchy sequence $\left\{z_{n}\right\}$ in $Z$, then $\left\{z_{n}\right\}$ converges uniformly to a function, for example, $z^{*}$, that is bounded and so $z^{*} \in Z$. Define $p\left(z_{1}, z_{2}\right)=e^{d\left(z_{1}, z_{2}\right) /\left(1+K d\left(z_{1}, z_{2}\right)\right)}$ for all $z_{1}, z_{2} \in Z$ and a fixed positive number $K$. Then $(Z, p)$ is a complete partial metric space.

We define $A, B: Z \rightarrow Z$ by

$$
\begin{aligned}
& A\left(z_{1}\right)(x)=\sup _{y \in Y_{1}}\left\{f(x, y)+G\left(x, y, z_{1}\left(\tau_{1}(x, y)\right)\right)\right\} \\
& B\left(z_{2}\right)(x)=\sup _{y \in Y_{1}}\left\{f(x, y)+H\left(x, y, z_{2}\left(\tau_{2}(x, y)\right)\right)\right\}
\end{aligned}
$$

for all $z_{1}, z_{2} \in Z$ and $x \in X_{1}$. Obviously, if the functions $f$ and $G, H$ are bounded then $A, B$ are well-defined.

We will prove the following theorems.

Theorem 12. Let

$$
\begin{gathered}
M\left(z_{1}, z_{2}\right)=\max \left\{p\left(z_{1}, z_{2}\right), p\left(z_{1}, A\left(z_{1}\right)\right),\right. \\
\left.p\left(z_{2}, B\left(z_{2}\right)\right), \frac{p\left(z_{1}, B\left(z_{2}\right)\right)+p\left(z_{2}, A\left(z_{1}\right)\right)}{2}\right\},
\end{gathered}
$$

where $p\left(z_{1}, z_{2}\right)=e^{d\left(z_{1}, z_{2}\right) /\left(1+K d\left(z_{1}, z_{2}\right)\right)}, K>0$. Suppose the following conditions are satisfied:

(H1) for all $\left(x, y, z_{1}, z_{2}\right) \in X_{1} \times Y_{1} \times Z \times Z$,

$$
\begin{aligned}
& \left|G\left(x, y, z_{1}\left(\tau_{1}(x, y)\right)\right)-H\left(x, y, z_{2}\left(\tau_{2}(x, y)\right)\right)\right| \\
& \quad \leq \ln \left(\max \left\{\phi\left(M\left(z_{1}, z_{2}\right)\right), p\left(z_{1}, z_{1}\right), p\left(z_{2}, z_{2}\right)\right\}\right),
\end{aligned}
$$

for all $x \in X_{1}$ and $y \in Y_{1}$, where $\phi:[0, \infty) \rightarrow[0, \infty)$ is upper-semicontinuous, such that $\phi(t)<t$ for any $t>0$. Then system (76) has a common bounded solution.

Proof. Let $\varepsilon$ be an arbitrary positive number, $x \in X_{1}$, and $z_{1}, z_{2} \in Z$. From (79), there exist $y_{1}, y_{2} \in Y_{1}$ such that

$$
\begin{aligned}
& A\left(z_{1}\right)(x)<f\left(x, y_{1}\right)+G\left(x, y_{1}, z_{1}\left(\tau_{1}\left(x, y_{1}\right)\right)\right)+\varepsilon, \\
& B\left(z_{2}\right)(x)<f\left(x, y_{2}\right)+H\left(x, y_{2}, z_{2}\left(\tau_{2}\left(x, y_{2}\right)\right)\right)+\varepsilon \\
& A\left(z_{1}\right)(x) \geq f\left(x, y_{2}\right)+G\left(x, y_{2}, z_{1}\left(\tau_{1}\left(x, y_{2}\right)\right)\right), \\
& B\left(z_{2}\right)(x) \geq f\left(x, y_{1}\right)+G\left(x, y_{1}, z_{2}\left(\tau_{2}\left(x, y_{1}\right)\right)\right),
\end{aligned}
$$

Then, from (82) and (85), it follows easily that

$$
\begin{aligned}
& A\left(z_{1}\right)(x)-B\left(z_{2}\right)(x) \\
& <G\left(x, y_{1}, z_{1}\left(\tau_{1}\left(x, y_{1}\right)\right)\right)+\varepsilon \\
& \quad-H\left(x, y_{1}, z_{2}\left(\tau_{2}\left(x, y_{1}\right)\right)\right) \\
& \leq\left|G\left(x, y_{1}, z_{1}\left(\tau\left(x, y_{1}\right)\right)\right)-G\left(x, y_{1}, z_{2}\left(\tau\left(x, y_{1}\right)\right)\right)\right| \\
& \quad+\varepsilon \\
& \leq \ln \left(\max \left\{\phi\left(M\left(z_{1}, z_{2}\right)\right), p\left(z_{1}, z_{1}\right), p\left(z_{2}, z_{2}\right)\right\}\right) \\
& \quad+\varepsilon,
\end{aligned}
$$

and, similarly, from (83) and (84) we have

$$
\begin{aligned}
& B\left(z_{2}\right)(x)-A\left(z_{1}\right)(x) \\
& \quad<\ln \left(\max \left\{\phi\left(M\left(z_{1}, z_{2}\right)\right), p\left(z_{1}, z_{1}\right), p\left(z_{2}, z_{2}\right)\right\}\right) \\
& \quad+\varepsilon .
\end{aligned}
$$

Therefore, we have

$$
\begin{aligned}
& \left|A\left(z_{1}\right)(x)-B\left(z_{2}\right)(x)\right| \\
& \quad<\ln \left(\max \left\{\phi\left(M\left(z_{1}, z_{2}\right)\right), p\left(z_{1}, z_{1}\right), p\left(z_{2}, z_{2}\right)\right\}\right) \\
& \quad+\varepsilon .
\end{aligned}
$$

We have

$$
\begin{aligned}
p( & \left.A\left(z_{1}\right), B\left(z_{2}\right)\right)=e^{d\left(A z_{1}, B z_{2}\right) /\left(1+K d\left(A z_{1}, B z_{2}\right)\right)} \\
& \leq e^{d\left(A z_{1}, B z_{2}\right)} \\
& \leq \max \left\{\phi\left(M\left(z_{1}, z_{2}\right)\right), p\left(z_{1}, z_{1}\right), p\left(z_{2}, z_{2}\right)\right\} e^{\varepsilon} .
\end{aligned}
$$

Since the above inequality does not depend on $x \in X_{1}$ and $\varepsilon>0$ is taken arbitrary, then we conclude immediately that

$$
\begin{aligned}
& p\left(A\left(z_{1}\right), B\left(z_{2}\right)\right) \\
& \quad \leq \max \left\{\phi\left(M\left(z_{1}, z_{2}\right)\right), p\left(z_{1}, z_{1}\right), p\left(z_{2}, z_{2}\right)\right\} .
\end{aligned}
$$

Then all the hypotheses of Theorem 3 are satisfied with $A, B$. Thus we deduce that $A, B$ have a common fixed point $z \in Z$. That is, the system of functional equations (76) has a common bounded solution. 


\section{Competing Interests}

The authors declare that they have no competing interests.

\section{Authors' Contributions}

All authors read and approved the final manuscript.

\section{Acknowledgments}

The authors are very grateful to Professor N. Mlaiki for sending his papers: "N. Mlaiki, A Partially Alpha-Contractive Principle, J. Adv. Math. Stud. Vol 7 (1), 121-126 (2014)" and "Thabet Abdeljawad, Kamal Abo Dayeh, Nabil Mlaiki, On Fixed Point Generalizations to Partial $b$-Metric Spaces, Journal of Computational Analysis and Application, vol. 19 (5) 883-891 (2015)," to us for helping us in modifying the present manuscript. Finally, this work was supported by the National Natural Science Foundation of China (11171286).

\section{References}

[1] S. G. Matthews, "Partial metric topology," Research Report 212, Department of Computer Science, University of Warwick, 1992.

[2] S. G. Matthews, "Partial metric topology," in Proceedings of the 8th Summer Conference on General Topology and Applications, vol. 728, pp. 183-197, Annals of the New York Academy of Sciences, 1994.

[3] I. Altun and A. Erduran, "Fixed point theorems for monotone mappings on partial metric spaces," Fixed Point Theory and Applications, vol. 2011, Article ID 508730, 10 pages, 2011.

[4] I. Altun, F. Sola, and H. Simsek, "Generalized contractions on partial metric spaces," Topology and Its Applications, vol. 157, no. 18, pp. 2778-2785, 2010.

[5] I. Altun and K. Sadarangani, "Corrigendum to 'Generalized contractions on partial metric spaces' [Topology Appl. 157 (2010) 2778-2785]," Topology and Its Applications, vol. 158, no. 13, pp. 1738-1740, 2011.

[6] S. Romaguera, "Fixed point theorems for generalized contractions on partial metric spaces," Topology and Its Applications, vol. 159, no. 1, pp. 194-199, 2012.

[7] T. Abdeljawad, E. Karapınar, and K. Taş, "A generalized contraction principle with control functions on partial metric spaces," Computers \& Mathematics with Applications, vol. 63, no. 3, pp. 716-719, 2012.

[8] T. Abdeljawad, E. Karapınar, and K. Taş, "Existence and uniqueness of a common fixed point on partial metric spaces," Applied Mathematics Letters, vol. 24, no. 11, pp. 1900-1904, 2011.

[9] R. H. Haghi, S. Rezapour, and N. Shahzad, "Be careful on partial metric fixed point results," Topology and Its Applications, vol. 160, no. 3, pp. 450-454, 2013.

[10] T. Abdeljawad, K. A. Dayeh, and N. Mlaiki, "On fixed point generalizations to partial b-metric spaces," Journal of Computational Analysis and Application, vol. 19, no. 5, pp. 883-891, 2015.

[11] D. Ilić, V. Pavlović, and V. Rakočević, "Some new extensions of Banach's contraction principle to partial metric space," Applied Mathematics Letters, vol. 24, no. 8, pp. 1326-1330, 2011.

[12] K. P. Chi, E. Karapınar, and T. D. Thanh, "A generalized contraction principle in partial metric spaces," Mathematical and Computer Modelling, vol. 55, no. 5-6, pp. 1673-1681, 2012.
[13] T. Abdeljawad and Y. Zaidan, "A phi-contraction principle in partial metric spaces with self-distance terms," Universal Journal of Mathematics and Mathematical Sciences, vol. 5, no. 2, pp. 133-147, 2014.

[14] N. Mlaiki, "A contraction principle in partial S-metric space," Universal Journal of Mathematics and Mathematical, vol. 5, no. 2, pp. 109-119, 2014.

[15] N. Mlaiki, "A partially alpha-contractive principle," Journal of Advanced Mathematical Studies, vol. 7, no. 1, pp. 121-126, 2014.

[16] T. Abdeljawad, "Coupled fixed point theorems for partially contractive mappings," Fixed Point Theory and Applications, vol. 2012, article 148, 2012.

[17] M. Asadi, E. Karapınar, and P. Salimi, "New extension of $p$ metric spaces with some fixed-point results on $M$-metric spaces," Journal of Inequalities and Applications, vol. 2014, p. 18, 2014.

[18] K. Abodayeh, N. Mlaiki, T. Abdeljawad, and W. Shatanawi, "Relations between partial metric spaces and M-metric spaces, Caristi Kirk's Theorem in M-metric type spaces," Journal of Mathematical Analysis, vol. 7, no. 3, pp. 1-12, 2016.

[19] N. Hussain, Z. Kadelburg, S. Radenovic, and F. R. Al-Solamy, "Comparison functions and fixed point results in partial metric spaces," Abstract and Applied Analysis, vol. 2012, Article ID 605781, 15 pages, 2012.

[20] N. Hussain, J. R. Roshan, V. Parvaneh, and A. Latif, "A unification of $G$-metric, partial metric and b-metric spaces," Abstract and Applied Analysis, vol. 2014, Article ID 180698, 14 pages, 2014.

[21] R. Bellman and E. S. Lee, "Functional equations in dynamic programming," Aequationes Mathematicae, vol. 17, no. 1, pp. 1$18,1978$.

[22] P. C. Bhakta and S. Mitra, "Some existence theorems for functional equations arising in dynamic programming," Journal of Mathematical Analysis and Applications, vol. 98, no. 2, pp. 348362, 1984. 


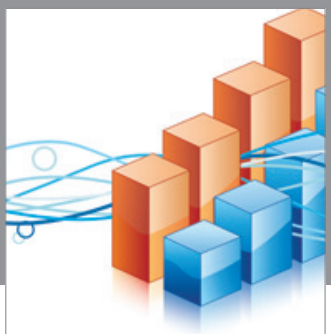

Advances in

Operations Research

vatem alat4

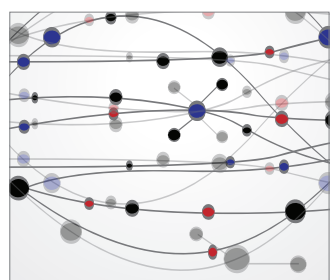

\section{The Scientific} World Journal
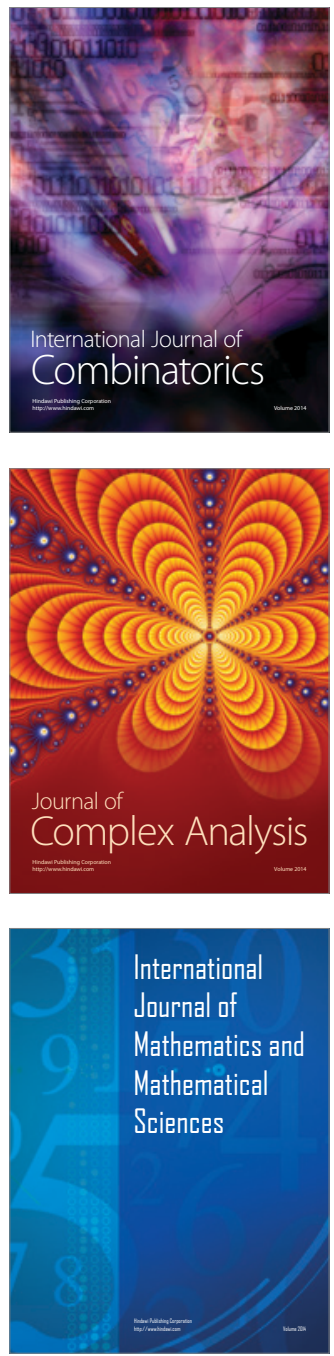
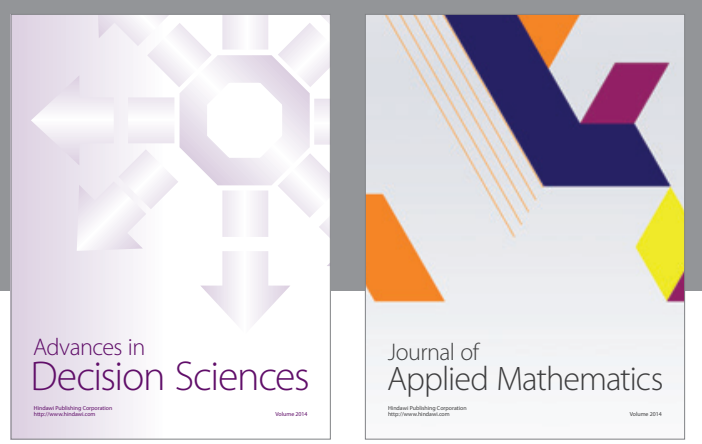

Algebra

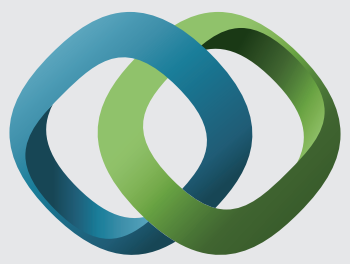

\section{Hindawi}

Submit your manuscripts at

http://www.hindawi.com
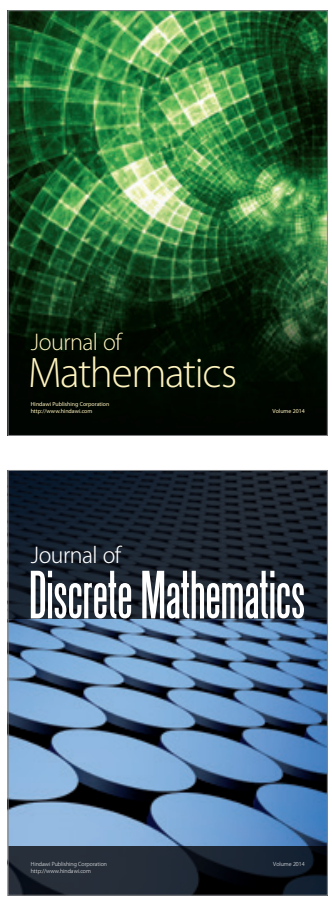

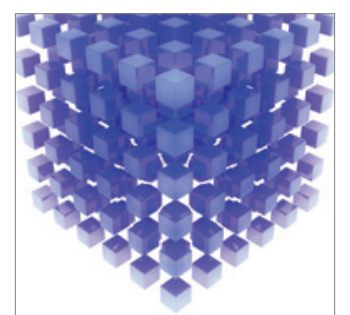

Mathematical Problems in Engineering
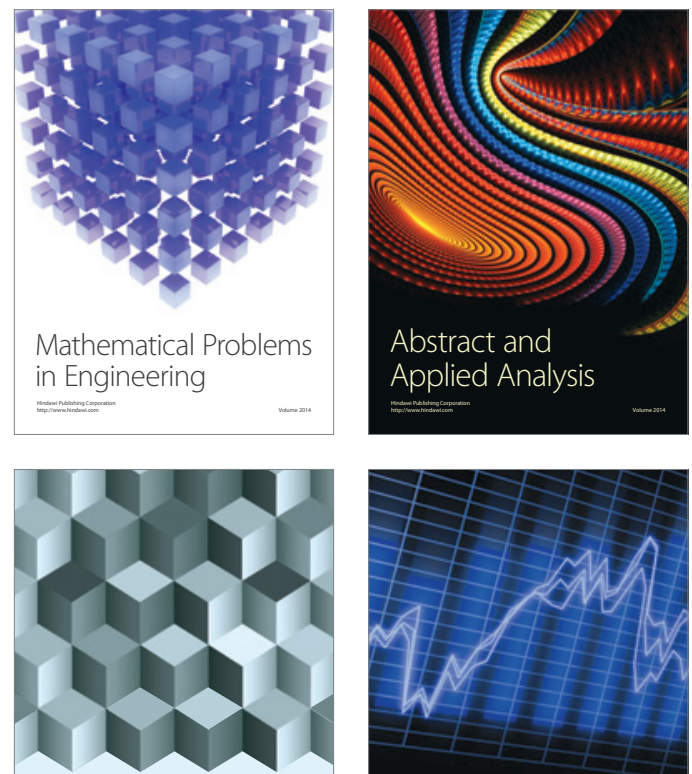

Journal of

Function Spaces

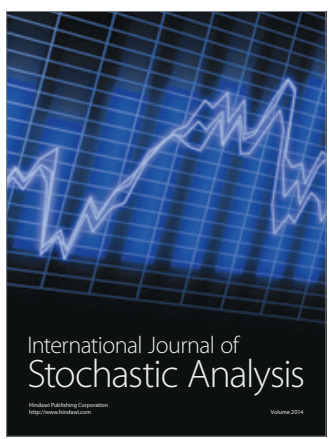

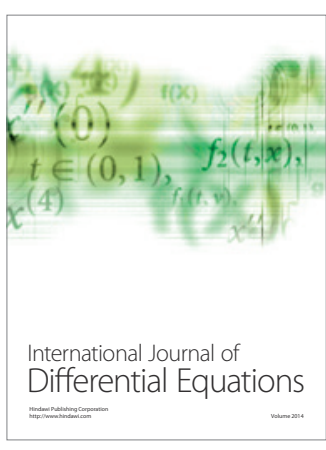
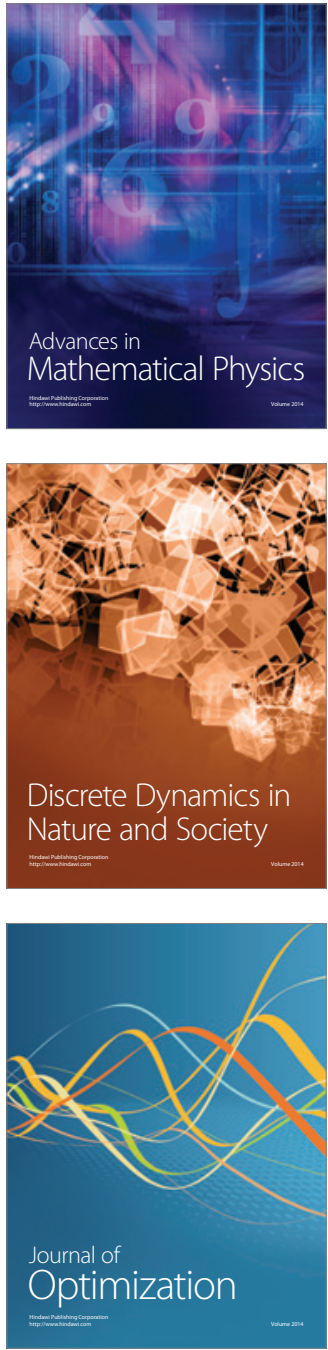